observatoires convenablement répartis en longitude, afin d'éviter les lacunes dues au mauvais temps.

Elle attire l'attention sur la nécessité de diffuser ces ursigrammes quotidiens suivant le même code, à des heures voisines, mais ne se recouvrant pas.

\title{
Commission 12. (RAdiation et SPECTROscopie solaires)
}

I. La Commission considère que la publication d'une table révisée du spectre solaire dans le plus court délai possible est de la plus haute importance pour les astronomes qui s'occupent de recherches sur le soleil et sur les spectres stellaires. Pour réduire le délai de publication, la Commission I2 recommande que la table révisée soit limitée à l'intervalle spectral encore inédit de $\lambda 3063$ à $\lambda 6600$, et que les longueurs d'onde soient données dans l'échelle de 1928. A cet effet, la Commission recommande que l'U.A.I. accorde une subvention de $£ 500$ ou de $\$ 2000$ pour la publication de la table solaire révisée.

\section{Commission i6. (OBservations Physiques des Planètes)}

I. La Commission décide de ne donner des nouveaux noms qu'à des formations lunaires importantes parmi celles qui n'ont pas encore été désignées.

Elle propose de donner le nom de Deslandres à la grande dépression rectangulaire située au sud du mur droit et qui contient les cratères Hell et Lexell.

2. La Commission recommande aux observateurs de la Lune de travailler uniquement sur des gabarits tracés au moyen de photographies à très grande échelle prises dans des conditions d'éclairement appropriées.

Elle recommande aux sections lunaires des associations astronomiques de s'entendre directement entre elles et avec les observatoires bien équipés pour l'obtention de telles photographies.

3. La Commission recommande aux observateurs qui étudient les colorations des planètes, d'utiliser des dispositifs corrigeant la dispersion atmosphérique, lorsque les observations sont faites à une faible hauteur.

\section{Commission I7. (Mouvement et Figure de LA LUNe)}

I. La Commission est unanime à estimer qu'il serait important d'entreprendre la réduction de l'ensemble des observations relatives à la libration de la lune par une méthode rigoureuse. L'exécution de cette grande tâche serait facilitée par la création d'un laboratoire mathématique international, qui serait une section de l'Observatoire International projeté.

2. La Commission est unanime à estimer que les travaux poursuivis par M. C. B. Watts (Washington) sur les irrégularités du limbe lunaire sont importants et méritent tout l'appui de la Commission.

3. La Commission est unanime à donner acte au 'Nautical Almanac Office' de ce que les prédictions d'occultations telles qu'elles sont calculées par ses soins sont fort utiles et contribuent au progrès de la branche de l'Astronomie dont s'occupe la Commission.

\section{Commission I8. (LongItUdes PAR TÉLÉGRAPHIE SANS FIL)}

I. Comme suite aux décisions prises lors de l'Assemblée Générale de Stockholm en I938, la Commission demande à l'Assemblée Générale que la publication déjà entreprise des Résultats de l’Opération mondiale des Longitudes de I933 soit liquidée aux moindres frais dans les conditions qui sont précisées dans le Rapport de son Président (voir 'Draft Reports', pp. 87-90) et qui correspondent à un partage exact des dépenses avec l'Association Internationale de Géodésie. 